\title{
Simultaneous use of magnetic method in localization of impalpable breast cancer and sentinel lymph nodes detection: initial experience
}

\author{
Kamil Pohlodek*,1, Martin Foltín, Iveta Mečiarová ${ }^{*}$ \& František Ondriaš² \\ ${ }^{1}$ Second Department of Gynecology \& Obstetrics, Faculty of Medicine, Comenius University of Bratislava, 82606 Bratislava, \\ Slovakia \\ ${ }^{2}$ Alpha Medical Pathology, Ltd, 84101 Bratislava, Slovakia \\ *Author for correspondence: kpohlodek@gmail.com
}

\begin{abstract}
Aim: In this study we used a new technology for localization of non-palpable breast tumors using a small steel marker in conjunction of sentinel nodes (SLNs) detection through injection of SPIO nanoparticles; both detected through a magnetic probe. Materials \& methods: Ten patients with biopsy-proven nonpalpable invasive breast carcinoma or premalignant lesions eligible for SLNs biopsy were enrolled in this study. Results: All tumors were removed with safe surgical margins. The mean nodal detection rate was 3.4 nodes per patient. No interferences in magnetic probe measurements due to the presence of both markers in the same breast were observed. Conclusion: Simultaneous use of the magnetic method in localization of impalpable breast tumors and SNs detection makes breast surgery convenient.
\end{abstract}

First draft submitted: 27 June 2018; Accepted for publication: 11 October 2018; Published online: 15 November 2018

Keywords: breast cancer $\bullet$ breast surgery $\bullet$ impalpable breast lesions • iron oxide nanoparticles $\bullet$ sentinel lymph nodes

Superparamagnetic iron oxide (SPIO) nanoparticles have been used in the past as contrast agents for magnetic resonance imaging (MRI). [1]. Moreover, SPIO have been recently used in breast cancer surgery for sentinel lymph node (SLN) detection $[2-8]$. Sienna $+{ }^{\circledR}$ is a brown solution containing carboxydextran-coated SPIO nanoparticles with a size of $59 \mathrm{~nm}$. After injection in the breast, Sienna+ drains through the lymphatics and accumulates in the SLNs. A handheld magnetic probe Sentimag ${ }^{\circledR}$ is used to identify the SLN by an acoustic signal and detection counts on display of the base unit. The SentiMAG multicentre trial [2] evaluated the magnetic technique against the standard (radioisotope alone or with blue dye) and concluded that the technique is a feasible method for SLN biopsy (SLNB) and that the identification rate is not inferior to the standard technique. The authors of the central-European SentiMag study [3] used the radiotracer and magnetic method in a parallel manner. They obtained convincing results that magnetic SLNB can be performed easily, safely and equivalently well in comparison to the radiotracer method. Further clinical trials followed [4-8] and all of them demonstrated the success of the magnetic method with SPIO in sentinel nodes $(\mathrm{SNs})$ detection in breast cancer patients.

Recently, another possibility has been presented for the use of magnetism in breast surgery. Magseed ${ }^{\circledR}$, a $5.0 \times 1.0 \mathrm{~mm}$ medical grade stainless steel - low nickel seed with magnetic detection property, was used as an alternative method for preoperative localization of impalpable breast cancer $[9,10]$. The seed is detectable using the Sentimag probe in the same way as the Sienna+ tracer in SLNs. For several years, the standard technique for intraoperative tumor localization of clinically occult tumors has been wire-associated localization. Here, a hook wire is placed into the tumor under ultrasound, $\mathrm{x}$-ray mammography or MRI guidance [11,12]. However, wire-based localization techniques are associated with disadvantages such as logistical challenges and migration issues. Radioactive technologies such as radio-guided occult lesion localization (ROLL) and radioactive seed localization (RSL) are used in some hospitals to overcome many of the disadvantages of wire localization, but they are connected with regulatory barriers and administrative burdens due to their radioactive manipulation [11,12].

Future Medicine 


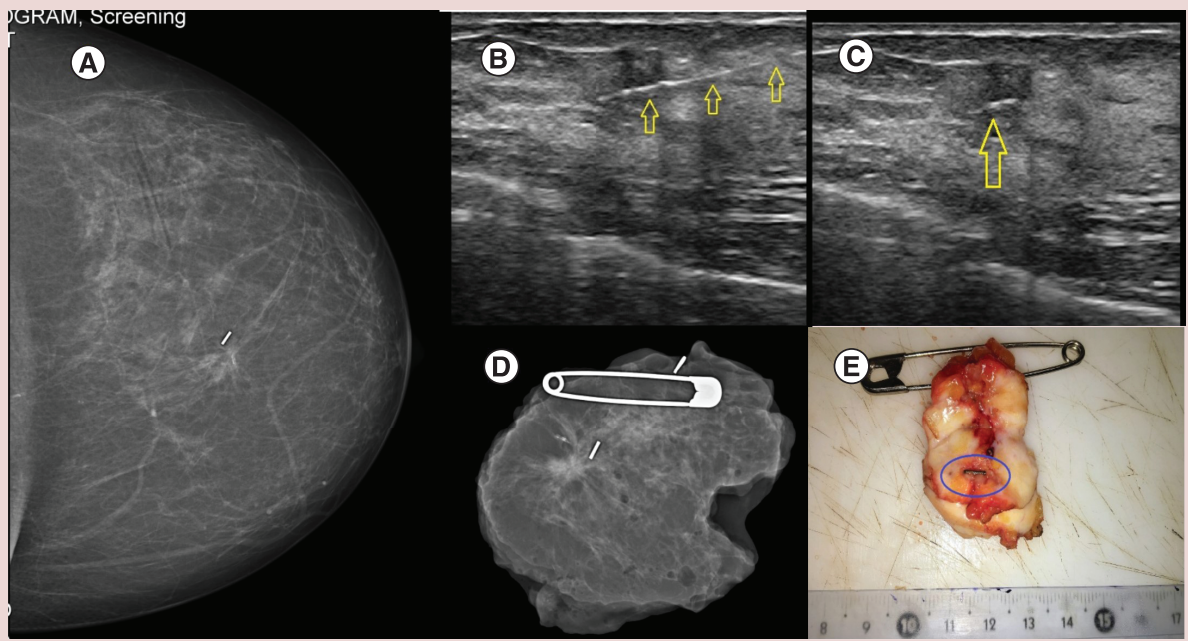

Figure 1. A nonpalpable infiltrating ductal carcinoma in the left breast marked with Magseed ${ }^{\circledR}$ in $x$-ray mammography (A). Insertion of Magseed under ultrasound guidance (B). The marker is seen in the middle of the lesion (C). Specimen radiography of the tumor with Magseed. The closing pin and the titanium clip on the upper pole of the sample serve as a guide for orientation of the specimen (D). Gross examination of the surgical specimen with inserted Magseed marker (E).

Magseed can be placed with 18-gauge needle deployment under local anesthetic into the center of the target lesions under ultrasound or mammographic guidance up to 30 days before the surgery [9]. The surgeon then uses the Sentimag device to confidently localize the Magseed marker in the breast. It is detectable from any orientation and offers guidance on distance to the seed. The aim of our study was to acquire initial experience in simultaneous use of the magnetic method in tumor localization and SLNs detection in breast cancer patients.

\section{Materials \& methods}

Study design

Ten patients were enrolled in this pilot, single-institutional, cohort study between April and May 2018 at the Breast unit of the 2nd Department of Gynecology and Obstetrics, Comenius University of Bratislava, Slovakia. Written informed consent was obtained from all patients. The inclusion criteria were patients with a core biopsy-proven early cT1 breast invasive carcinoma or premalignant breast lesions (ductal carcinoma in situ [DCIS], atypical ductal hyperplasia $[\mathrm{ADH}]$ ) with clinically (palpation + ultrasound) negative axilla (cN0) eligible for SLNB procedure for which breast conserving surgery was planned. The exclusion criteria were a pacemaker or implanted device in the chest wall, iron/nickel allergy, Sienna allergy and pregnancy or lactation. The study was approved by the Comenius University and University Hospital of Bratislava Ethics Committee.

\section{Localization procedures}

All localization markers (Magseed ${ }^{\circledR}$, Endomagnetics Ltd, Cambridge, UK) were inserted with 18-gauge needle deployment under local anesthesia into the center of the target lesions under ultrasound guidance on the same day prior to surgery. Ipsilateral two-view mammography (mediolateral and craniocaudal views) were performed immediately after the localization procedure to document the marker position (Figure 1).

\section{Surgical procedures}

In patient with preoperative core-needle biopsy proven infiltrating carcinoma and/or DCIS lymphatic mapping and SLNB were the initial surgical procedures. After induction of general anaesthesia, $2.0 \mathrm{ml}$ of SPIO nanoparticles (Sienna+, Endomagnetics Ltd, Cambridge, UK), diluted to $5.0 \mathrm{ml}$ with physiological saline solution, was injected into the subareolarly interstitial tissue at least $20 \mathrm{~min}$ before SLNB, followed by $5 \mathrm{~min}$ massage of the breast to promote migration of the magnetic tracer. Before skin incision, count numbers on the skin, at the injection site and in the axillary area (hot spots) were measured with the 2 nd generation magnetic probe (Sentimag, Endomagnetics 

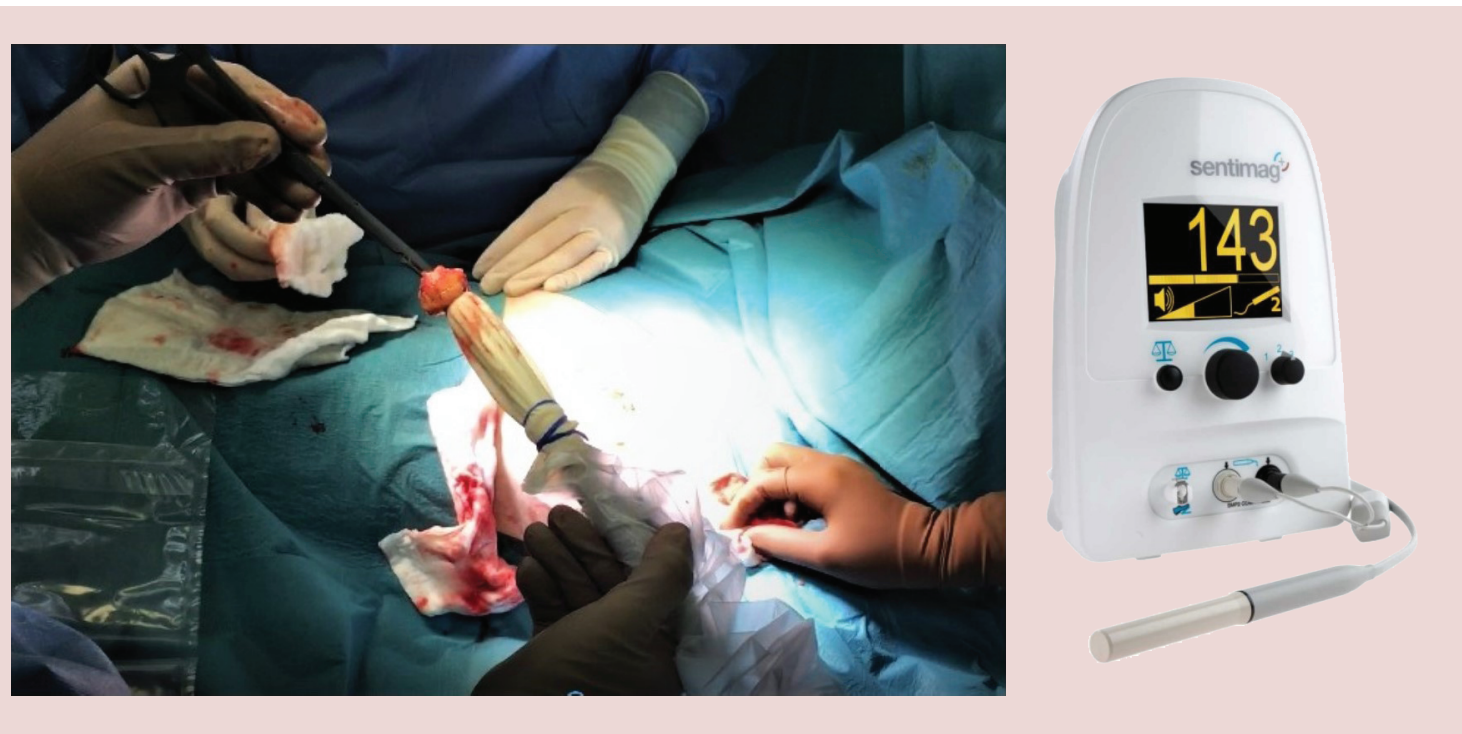

Figure 2. Surgeon uses the Sentimag ${ }^{\circledR}$ probe to localize the Magseed ${ }^{\circledR}$ marker in the surgical specimen (on the left). The probe generates an alternating magnetic field that transiently magnetizes the iron oxide nanoparticles within the seed and/or within the sentinel nodes. The Sentimag base unit (on the right) displays a numerical count and produces an audio tone depending to the distance of the seed and/or sentinel nodes from the detector probe.

Ltd, Cambridge, UK). Magnetic counts were registered also intraoperatively after the skin incision, in situ and $e x$ vivo in the SLNs and in the remaining axilla. All SLNs were excised until the counts were lower than $10 \%$ of the highest count or a maximum of four nodes per patient were removed. All SLNs were examined intraoperatively by frozen section procedure. In patients with metastases in SLNs, additionally axillary level $1+2$ lymphadenectomy was provided. All SLNs (and axillary non-SLNs, alternatively) were postoperative histologically examined for metastases through serial sectioning and immunohistochemistry. SLNB was omitted in one patient with diagnosis of $\mathrm{ADH}$.

After SLNs detection and excision, the Magseed was localized in the breast using the Sentimag probe. The signal hot spot (maximum recorded detector count) was signed on the skin prior to skin incision. Breast conserving surgical procedures (lumpectomy, segmentectomy or quadrantectomy) appropriate to tumor diameter with adequate resection margins and to whole breast volume, were provided. After tumor excision, Sentimag probe was repeated used to confirm the presence of Magseed in the surgical specimen (Figure 2). Two-view specimen radiography was performed during surgery to confirm complete tumor resection with Magseed and with sufficient margins. All surgical specimens were than inked, fixed in formalin and examined through serial sectioning and immunohistochemistry.

\section{Pathology}

All SLNs were examined intraoperatively by frozen section. SLNs with clearly presented metastases were determined as positive and additional axillary dissection was provided. All lymph nodes were than postoperatively evaluated for micrometastases in formalin-fixed embedded serial sections using hematoxylin/eosin staining and immunohistochemistry with cytokeratin AE1/AE3 stains. The surgical specimens were fixed in $10 \%$ neutral-buffered formalin for $24 \mathrm{~h}$. The fixed specimens were examined through serial sectioning. The sections were then stained with hematoxylin and eosin. Immunohistochemistry was used for evaluation of estrogen, progesteron and HER2 receptors, Ki67 proliferation index and myoepithelial cells integrity assessment. Specimens with no ink on tumor were evaluated as specimens with negative surgical margins, according to the current guidelines [13].

\section{Results}

The mean age of our group of patients was 48.7 years and the mean BMI was 24.9. Tumor characteristics of all surgically treated impalpable breast lesions are shown in Table 1 . The mean diameter of the largest lesions was $10.3 \mathrm{~mm}$ (from 4 to $15 \mathrm{~mm}$ ) in breast imaging ( $\mathrm{x}$-ray mammography and/or ultrasound), and $15.3 \mathrm{~mm}$ (from 6 to 


\begin{tabular}{|c|c|c|c|c|c|c|c|c|c|}
\hline Patient ID & $\begin{array}{l}\emptyset \text { lesion } \\
\text { (imaging) mm }\end{array}$ & $\begin{array}{l}\emptyset \text { lesion } \\
\text { (histology) } \\
\mathrm{mm}\end{array}$ & Tumor typing & Tumor grading & ER/PR (\%/\%) & HER2 $(0 /+)$ & $\begin{array}{l}\text { Resection } \\
\text { margins }\end{array}$ & SLN status & ALN status \\
\hline 1 & 12 & 15 & DCIS & 2 & $95 / 75$ & 0 & Negative & $0 / 2$ & - \\
\hline 2 & 4 & 6 & ADH & - & - & - & Negative & - & - \\
\hline 3 & 9 & 11 & IDC & 2 & $95 / 70$ & 0 & Negative & $0 / 2$ & - \\
\hline 4 & 8 & 12 & IDC & 2 & $100 / 100$ & $1+$ & Negative & $0 / 3$ & - \\
\hline 5 & 12 & 16 & IDC & 2 & $99 / 30$ & 0 & Negative & $0 / 6$ & - \\
\hline 6 & 5 & 15 & DCIS & 2 & $95 / 95$ & 0 & Negative & $0 / 4$ & - \\
\hline 7 & 11 & 15 & IDC & 2 & $99 / 70$ & $2+$ & Negative & $0 / 5$ & - \\
\hline 8 & 15 & 17 & IDC & 3 & $90 / 60$ & 0 & Negative & $2 / 3$ & $4 / 11$ \\
\hline 9 & 15 & 25 & IDC & 2 & $90 / 0$ & 0 & Negative & $4 / 5$ & $6 / 25$ \\
\hline 10 & 12 & 21 & DCIS & 3 & $5 / 5$ & $3+$ & Negative & $0 / 4$ & - \\
\hline
\end{tabular}

$\varnothing$ lesion: The largest diameter of the breast lesion in breast imaging and in histological examination in $\mathrm{mm}$.

ADH: Atypical ductal hyperplasia; ALN status: Number of axillary lymph nodes with metastases/number of examined axillary lymph nodes; DCIS: Ductal carcinoma in situ; IDC: Infiltrating ductal carcinoma; Resection margins: Margin status of examined surgical specimens (negative = no ink on tumor); SLN status: Number of sentinel lymph nodes with metastases/number of examined sentinel lymph nodes.

$25 \mathrm{~mm}$ ) in histopathology. In six cases infiltrating breast carcinoma was diagnosed. Three patients presented DCIS and another one $\mathrm{ADH}$ in definitive diagnoses from surgical specimens. A residual impalpable tumor after six cycles of neoadjuvant chemotherapy was localized in one patient. All impalpable lesions were precisely localized with Magseed and accurately detected in the breast with Sentimag system. The seed could be successfully recognized by pathologist through grossly examination in all specimens. All tumors were removed with safe surgical margins through the first operation and no additional re-excision of margins was needed. The mean nodal detection rate (the proportion of SLNs detected per patient) was 3.4 (from 2 to 6). Only two of all our patients presented metastases in SLNs and additional axillary dissection was provided. In both cases, further metastases in axillary non-SLNs were found. No interference in magnetic probe measurements through presence of both Magseed and Sienna markers in the same breast was observed. The mean depth of Magseed insertion in the breast was $2.4 \mathrm{~cm}$ (from 1.5 to $5.8 \mathrm{~cm}$ ). No complications or adverse events related to either the seed placement or to the surgery were recorded. No migration of Magseed in the breast from the time of its placement to the time of surgery was observed.

\section{Discussion}

Sienna+ particles are engineered to be uniform and small enough for rapid migration through lymphatics, yet large enough to be mechanically filtered by the SLNs. Sienna+ injection can be applied by surgeon in operation theater at least 20 min prior to surgery. Using a highly sensitive Sentimag probe, the surgeon can detect very small quantities of Sienna in the SLNs. The tracer is also dark in color that offers the surgeon a visual confirmation and helps eliminate the need for blue dyes. The method SPIO nanoparticles that can be detected using a magnetometer was in prospective randomized clinical trials established to be not inferior to SLNB procedure with the standard combined technique (radionuclide plus blue dye) [2-8].

Three systematic reviews and/or meta-analyses of studies were recently performed by Karakatsanis et al. [8], Zada et al. [14] and Teshome et al. [15] to assess the performance and utility of the magnetic method in comparison to the standard technique. All the authors concluded that the magnetic SLNB technique was noninferior to the standard technique, had a high identification rate and a significantly higher lymph node retrieval rate. In our department, about 120 breast cancer patients are surgically treated per year. The Sienna+/Sentimag system is helping us in SLNs detection for the last 5 years.

For breast-conserving surgery of small, nonpalpable tumors, an accurate tumor localization is essential. For several years, the standard technique for intraoperative tumor localization of clinically occult tumors has been wire-guided localization (WGL), in which a hook wire is placed into the tumor under ultrasound, $\mathrm{x}$-ray mammography or MRI guidance. However, the WGL procedure has been criticized for the last few years. Key disadvantages of WGL include the presence of a foreign object in the specimen for pathological assessment, possible wire transection, wire migration before or during surgery, patient distress and discomfort and pneumothorax. Another significant disadvantage of WGL is that the guide-wire does not provide a clear 3D perspective on the tumor [11,12]. 
This has led to the development of other guidance techniques, including ROLL and RSL. In ROLL technique, a nonspecific radioisotope is injected into the tumor under stereotactic or ultrasonographic guidance. The exact localization of the primary tumor can be assessed intraoperatively by use of a handheld gamma probe. After resection of the primary lesion, the probe also can be used to search for SLNs. It can be performed prior to the day of surgery and the surgeon can accurately localize the device in theater using a handheld gamma probe, similarly as in ROLL procedure. Both RSL and ROLL are equally reliable to wire localization [12], but they suffer from high regulatory barriers and administrative burdens due to their radioactive manipulation.

The new magnetic method is an alternative of localizing breast lesions. Magseed, a small marker containing SPIO nanoparticles has been used for localization of impalpable breast lesions. Harvey et al. [9] and Price et al. [10] published recently their initial clinical experience with this method. Both studies conclude that Magseed is an effective and accurate means of preoperative breast lesion localization. In the study by Harvey et al. [9], 29 Magseed devices were placed into the breasts of 28 patients, 24 under ultrasound guidance and five under stereotactic (mammographic) guidance. One patient had bilateral seed placement. 27 of 29 Magseeds were placed directly in the target lesion and all seed devices were retrieved. There were no complications or adverse events recorded related to either the seed placement or to the surgery. Magseed is currently available in the USA and Canada, and in Europe, Middle East and Africa (EMEA).

We report our first experience with simultaneous localization of impalpable breast tumors and SLNs detection with SPIO nanoparticles through the magnetic method. All our first ten patients with impalpable breast tumors could be accurately localized with the magnetic method. No interferences between SPIO signals from Magseed and from Sienna + tracer during magnetic probe measurements were observed. All carcinomas were excised through the first operation and no re-excision of surgical margins was needed. This technology would make tumor localization and SLNB procedure possible in areas where radioisotope availability is limited or a nuclear medicine department is unavailable.

\section{Conclusion}

To the best of our knowledge, this is the first feasibility report describing the clinical experience of the simultaneous use of the Magseed and Sienna/Sentimag system. All our first ten patients with impalpable breast tumors could be accurately localized with the magnetic method.

No interferences between SPIO signals from Magseed and from Sienna+ tracer during magnetic probe measurements were observed. All carcinomas were excised through the first operation and no re-excision of surgical margins was needed. Magnetic seeds are a feasible and safe method of breast lesion localization. They can be accurately placed, do not migrate and are detectable at all depths of breast tissue. Using Magseed in combination with Sienna + magnetic tracer for SLNs detection makes breast conserving surgery convenient and entirely independent of radioactive methods.

\section{Future perspective}

SLNB is currently the standard surgical procedure for staging of the clinically negative axilla in breast cancer patients. Detection of the SLN is based on the use of a radioactive tracer, most often in combination with a vital dye. The use of an isotope depends on access to the nuclear medicine department, which in some hospitals can mean logistical problems. Recent literature reports that only $60 \%$ of patients in developed countries have access to the procedure, with this figure dropping down to $5 \%$ in China and even lower in the rest of the world [8]. SLNB using SPIO nanoparticles is a novel method in breast cancer surgery. Several studies have verified the noninferiority of SPIO compared with the standard use of radioisotope $99 \mathrm{mTc}$ with or without blue dye [2-8]. Insertion of Magseed, a stainless steel - low nickel seed with magnetic detection property, into nonpalpable breast lesions can allow the surgeon to reliably localize and remove tumors and simultaneously detect the SLNs using the Sentimag system. Using Magseed in combination with Sienna+ magnetic tracer for SLNs detection can in the future make breast conserving surgery convenient, especially in hospitals where the radionuclide method is unavailable.

Financial \& competing interests disclosure

The study was funded by Sysmex Europe, GmbH, 22848 Norderstedt, Germany. The authors received no financial support for the research, authorship and/or publication of this article. The authors have no other relevant affiliations or financial involvement with any organization or entity with a financial interest in or financial conflict with the subject matter or materials discussed in the manuscript apart from those disclosed.

No writing assistance was utilized in the production of this manuscript. 
Summary points

- Breast conserving therapy with sentinel lymph nodes (SLNs) biopsy is currently standard surgical procedure in patients with early breast cancer.

- Through screening mammography, most of the early breast carcinomas present as nonpalpable lesions, which are usually localized through hook wires.

- Superparamagnetic iron oxide (SPIO) nanoparticles possess unique properties, which make them highly attractive for medical applications.

- The use of SPIO nanoparticles in surgery has mainly been focused on their role in the identification of metastatic lymph node involvement.

- Sienna $+{ }^{\circledR}$ is a brown solution containing carboxydextran-coated SPIO nanoparticles with a size of $59 \mathrm{~nm}$. After injection in the breast, it drains through the lymphatics and accumulates in the SLNs.

- Magseed ${ }^{\circledR}$, a $5.0 \times 1.0 \mathrm{~mm}$ medical grade stainless steel - low nickel seed with magnetic detection property, is used as an alternative method for preoperative localization of nonpalpable breast cancer.

- A handheld magnetic probe is used to identify SLNs and Magseed by an acoustic signal and detection counts on display on the base unit (Sentimag ${ }^{\circledR}$ ).

- Ten patients with core biopsy-proven nonpalpable breast lesions were enrolled in this pilot study with simultaneous use of Sienna+ and Magseed in breast cancer surgery.

- All impalpable lesions were precisely localized with Magseed and accurately detected in the breast with Sentimag system. All lesions were then excised with safe surgical margins.

- Using Magseed in combination with Sienna+ magnetic tracer for SLNs detection makes breast conserving surgery convenient and entirely independent of radioactive methods.

\section{Author contributions}

K Pohlodek performed the patient's examination, breast imaging, interventional breast procedures, breast surgery and analyzed and interpreted the patient data regarding the disease. $\mathrm{K}$ Pohlodek was a major contributor in writing the manuscript. M Foltín performed interventional breast procedures and breast surgery. I Mečiarová performed the histological examination of the core needle biopsy, surgical specimens and analyzed and interpreted the patient data regarding the histology. F Ondriaš assisted with the histological findings of the surgical specimens. The datasets used and/or analyzed during the current study are available from the corresponding author on reasonable request.

Ethical conduct of research

The study was approved by the Comenius University and University Hospital of Bratislava Ethics Committee. Written informed consent was obtained for patient participation and for the publication of all associated data and images. Written informed consent was obtained from patients for the publication of all associated data and images.

Open access

This work is licensed under the Attribution-NonCommercial-NoDerivatives 4.0 Unported License. To view a copy of this license, visit http://creativecommons.org/licenses/by-nc-nd/4.0/

\section{References}

Papers of special note have been highlighted as: $\bullet$ of interest; $\bullet \bullet$ of considerable interest

1. Wang YX, Idée JM. A comprehensive literatures update of clinical researches of superparamagnetic resonance iron oxide nanoparticles for magnetic resonance imaging. Quant. Imaging Med. Surg. 7, 88-122 (2017).

2. Douek M, Klaase J, Monypenny I et al. Sentinel node biopsy using a magnetic tracer versus standard technique: the SentiMAG multicentre trial. Ann. Surg. Oncol. 21, 237-245 (2014).

- A total of 160 women with breast cancers scheduled for SLNB, were recruited from seven centers in The United Kingdom and the Netherlands. SLNB was undertaken after administration of both the magnetic and standard tracers (radioisotope with or without blue dye).

3. Thill M, Kurylcio A, Weletr R et al. The central-European SentiMag study: sentinel lymph node biopsy with super paramagnetic iron oxide (SPIO) vs radioisotope. Breast 23, 175-179 (2014).

- A prospective, multicentre and multinational, non-randomized, paired equivalence study including 150 patients. $99 \mathrm{mTc}$ was compared with the magnetic technique, using SPIO (Sienna $+{ }^{\circledR}$ ) for localization of SLNs.

4. Rubio IT, Diaz-Botero S, Esgueva A et al. The superparamagnetic iron oxide is equivalent to the Tc99 radiotracer method for identifying the sentinel lymph node in breast cancer. Eur. J. Surg. Oncol. 41, 46-51 (2015). 
- A prospective, non-randomized trial. SLNB procedure was performed for 120 patients using the radioisotope (Tc99) and the SPIO tracer.

5. Ghilli M, Carretta E, Di Filippo F et al. The superparamagnetic iron oxide tracer: a valid alternative in sentinel node biopsy for breast cancer treatment. Eur. J. Cancer Care doi:10.1111/ecc.12385 (2017) (Epub ahead of print).

- A paired, prospective, multicentre study to evaluate the non-inferiority of SentiMag ${ }^{\circledR}$ vs. 99m Tc in SLNB in 193 breast cancer patients

6. Houpeau JL, Chauvet MP, Guillemin F et al. Sentinel lymph node identification using superparamagnetic iron oxide particles versus radioisotope: the French Sentimag feasibility trial. J. Surg. Oncol. 113, 501-507 (2016).

- The French prospective, multicentric paired trialusing SPIO nanoparticles in comparison to the standard technique (isotopes + blue dye) for SLNs detection in 115 breast cancer patients

7. Piñero-Madrona A, Torro-Richart JA, de Leon-Carrillo JM, et al. on behalf of the Grupo de Estudios Senologicos de la Sociedad Española de Patologia Mamaria (SESPM). Superparamagnetic iron oxide as a tracer for sentinel node biopsy in breast cancer: a comparative non-inferiority study. Eur. J. Surg. Oncol. 41, 991-997 (2015).

- Prospective, randomized trials for comparison of SPIO nanoparticles versus technetium Tc99 and patent blue in the detection of SNs in 181 patients with breast cancer.

8. Karakatsanis A, Christiansen PM, Fischer L et al. The Nordic SentiMag trial: a comparison of super paramagnetic iron oxide (SPIO) nanoparticles versus $\mathrm{Tc} 99$ and patent blue in the detection of sentinel node $(\mathrm{SN})$ in patients with breast cancer and a meta-analysis of earlier studies. Breast Cancer Res. Treat. 157, 281-294 (2016).

- Prospective, multicentre trial for comparison of SPIO nanoparticles versus technetium Tc99 and patent blue in the detection of SNs in 206 patients with breast cancer and a meta-analysis of earlier studies

9. Harvey JR, Lim Y, Murphy J et al. Safety and feasibility of breast lesion localization using magnetic seeds (Magseed): a multicentre, open-label cohort study. Breast Cancer Res. Treat. 169, 531-536 (2018).

-. A two-center, open-label cohort study to assess the feasibility and safety of Magseed localization of breast lesions in 28 patients.

10 Price ER, Khoury AL, Esserman LJ et al. Initial clinical experience with an inducible magnetic seed system for preoperative breast lesion localization. Am. J. Roentgenol. 4, 913-917 (2018).

-. All of the 73 Magseeds were successfully placed and all were successfully retrieved intraoperatively in this study

11 Chan BKY, Wiseberg-Firtell JA, Jois RHS et al. Localization techniques for guided surgical excision of nonpalpable breast lesions. Cochrane Database Syst. Rev. 12, CD009206 (2015).

12 Hayes MK. Update on preoperative breast localization. Radiol. Clin. N. Am. 55, 591-603 (2017).

13. Brouwer de Koning SG, Vrancken Peeters MTFD, Jóźwiak K et al. Tumor resection margin definitions in breast-conserving surgery: Systematic review and meta-analysis of the current literature. Clin. Breast Cancer 18(4), e595-e600 (2018).

14. Zada A, Peek MCL, Ahmed M et al. Meta-analysis of sentinel lymph node biopsy in breast cancer using the magnetic technique. $B r$. J. Surg. 11, 1409-1419 (2016).

15. Teshome $\mathrm{M}$, Wei $\mathrm{C}$, Hunt $\mathrm{KK}$ et al. Use of a magnetic tracer for sentinel lymph node detection in early-stage breast cancer patients: a meta-analysis. Ann. Surg. Oncol. 23, 1508-1514 (2016). 
(

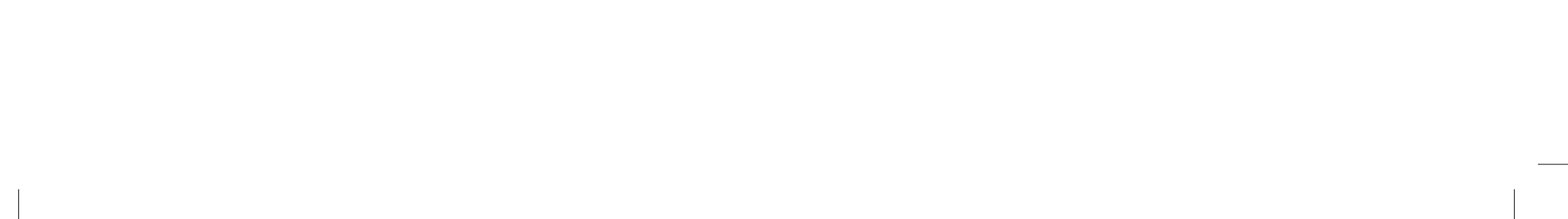

